\title{
DURÉE DU CYCLE DE L'ÉPITHÉLIUM SÉMINIFÈRE DU SINGE CRABIER, MACACA FASCICULARIS
}

\author{
D.-C. DANG \\ Station centrale de Physiologie animale, \\ Centre national de Recherches zootechniques, I. N. R. A., \\ 78 - Jouy-en-Josas \\ Laboratoire d'Éthologie comparée, C. N. R. S., \\ 91 - Saint-Chéron
}

\section{RÉSUMÉ}

Le marquage des cellules germinales par injection de thymidine tritiée a été utilisé pour estimer la durée du cycle de l'épithélium séminifère de Macaca fascicularis. Cette durée est de 9,3 jours et elle n'est pas affectée par l'hémicastration.

La durée du cycle de l'épithélium séminifère de l'Homme, r6 jours, (Heller et Clermont, 1963) est la plus longue si on la compare avec celle des autres Mammifères déjà étudiés par la même méthode. Ce caractère semble être propre aux Hominiens et non aux Primates dans leur ensemble puisque la durée du cycle de Macaca fascicularis est inférieure à celle du Rat, I2 jours (Clermont et al., I959) et du Taureau, I3,5 jours (Hochereau et al., I964).

\section{INTRODUCTION}

Au point de vue histologique si le cycle de l'épithélium séminifère a été bien étudié et décrit chez plusieurs Mammifères, la durée du cycle n'a été que peu étudiée. Pour les Primates nous n'en possédons que deux informations. La première étude a été faite par ARSENIEVA et al. (I96I). Par la méthode d'irradiation ces auteurs ont trouvé une durée du cycle du Macaque rhésus : Macaca mulatta, de I0,5 jours. HELLER et CIERMONT ( 1963 ) par marquage à l'aide de la thymidine tritiée ont déterminé chez l'Homme une durée de 16 jours, durée qui est la plus longue si on la compare avec celle des autres Mammifères déjà étudiés. Dans cette étude nous nous proposons d'estimer la durée dì cycle d'une autre espèce de Primate, le Macaque crabier, Macaca fascicularis. 


\section{MÉTHODE D'ÉTUDE}

Pour déterminer la durée du cycle de l'épithélium séminifère nous avons utilisé la méthode de marquage des cellules germinales à l'aide de la thymidine tritiée. La fréquence relative de chaque stade du cycle de l'épithélium séminifère représente sa durée relative par rapport à la durée totale du cycle (LeBlond et Clermont, 1952). De ce fait en suivant l'évolution du front de marquage au cours des stades successifs du cycle après un temps donné nous pouvons calculer la durée du cycle. Les fréquences relatives des huit stades du cycle de l'épithélium séminifère de Macaca fascicularis sont représentées sur la figure (DANG, I97I).

\section{MATÉRIEL ET TECHNIQUES}

Quatre animaux pubères, âgés de 6 à $\mathbf{1} 3$ ans, élevés au laboratoire, en bonne santé, ont été utilisés pour cette étude. L'hémicastration et la castration ont été faites suivant le tableau ci-après.

L'injection de thymidine tritiée a été pratiquée sur l'animal anesthésié par inhalation de protoxyde d'azote selon deux techniques :

\section{A. - Injection intratesticulaire}

Technique employée par Heller et Clermont (r963) chez l'Homme. roo $\mu \mathrm{Ci}$ de thymidine tritiée (C E A, activité spécifique $9 \mathrm{Ci} / \mathrm{mM}$ ) diluées dans $2 \mathrm{ml}$ de sérum physiologique sont injectées directement dans le testicule à travers le scrotum à une profondeur de $1,5 \mathrm{~cm}$ et à trois endroits régulièrement espacés et situés sur le côté opposé à l'emplacement de l'épididyme.

TABLEAU I

Plan expérimental

\begin{tabular}{|c|c|c|c|}
\hline \multirow{2}{*}{ Animaux } & \multirow{2}{*}{ Age } & \multicolumn{2}{|c|}{ Prélèvements des testicules } \\
\hline & & Gauche & Droit \\
\hline$\sigma_{1}$ & $12-13$ ans & $5-3-68$ à $17 \mathrm{~h}$ & $\begin{array}{c}25-4-68 \text { à } 18 \mathrm{~h} \\
30^{\prime} \text { après injection intraartérielle } \\
\text { de thymidine- }{ }^{-3} \mathrm{H}\end{array}$ \\
\hline$\delta_{2}$ & $9-10$ ans & $\begin{array}{l}14-5-68 \text { à } 17 \mathrm{~h} \\
453 \mathrm{~h} \text { après injection intratesti- } \\
\text { culaire de thymidine- }{ }^{8} \mathrm{H}\end{array}$ & $\begin{array}{l}\quad 7-5.68 \text { à } 16 \mathrm{~h} 30^{\prime} \\
284 \mathrm{~h} 30^{\prime} \text { après injection intra- } \\
\text { testiculaire de thymidine- }{ }^{3} \mathrm{H}\end{array}$ \\
\hline$\delta_{5}$ & 6.7 ans & $3-2-69$ à $12 \mathrm{~h}$ & $\begin{array}{c}\quad 10-2-69 \text { à } 14 \mathrm{~h} 30^{\prime} \\
622 \mathrm{~h} \mathbf{3 0}^{\prime} \text { après injection intra- } \\
\text { artérielle de thymidine }-{ }^{3} \mathrm{H}\end{array}$ \\
\hline$\delta_{7}^{*}$ & $6-7$ ans & $\begin{array}{c}25-11-69 \text { à } 16 \mathrm{~h} \quad 15^{\prime} \\
45^{\prime} \text { après injection intraartérielle } \\
\text { de thymidine- }{ }^{3} \mathrm{H}\end{array}$ & $\begin{array}{c}17-11-69 \text { à } 15 \mathrm{~h} 15^{\prime} \\
45^{\prime} \text { après injection intraartérielle } \\
\text { de thymidine- }{ }^{3} \mathrm{H}\end{array}$ \\
\hline
\end{tabular}




\section{B. - Injection intra-artérielle}

Selon la technique proconisée par Hochereau et al. (1964) pour le Taureau. $250 \mu \mathrm{Ci}$ de la même thymidine tritiée diluée dans $2 \mathrm{ml}$ de sérum physiologique sont injectés très lentement dans l'artère testiculaire dégagée après ouverture longitudinale du scrotum, de la tunique fibreuse et de la tunique vaginale.

Après hémicastration ou castration, des petits cubes du parenchyme testiculaire sont fixés au Bouin-Hollande, déshydratés et inclus dans la paraffine. Les coupes de $6 \mu$ d'épaisseur, après coloration à 1'APS-Feulgen sont recouvertes d'émulsion pelliculable KoDAK AR ro (KOPRIWA et Leblond, ig62), exposées de 28 à $4^{\circ}$ jours et révélées selon les procédés classiques à l'aide d'un révélateur à l'amidol.

\section{RÉSULTATS}

\section{A. - Estimation de la durée du cycle}

$30^{\prime}-45^{\prime}$ après injection de la thymidine radioactive, dans le testicule de l'animal normal $\left(\hat{\sigma}_{7}\right)$ ou de l'animal hémicastré $\left(\hat{\sigma}_{1}\right)$ on trouve outre des spermatogonies marquées quand elles sont à la phase de synthèse d'ADN des spermatocytes I préleptotènes et leptotènes marqués au début du stade I du cycle (fig. A). Aucun spermatocyte du stade 2 n'est marqué. Les spermatocytes I leptotènes sont donc les cellules germinales marquées les plus avancées dans le cycle spermatogénétique. Elles représentent le front de radioactivité qui nous sert comme point de départ ou point de repère pour calculer la durée du cycle.

$284 \mathrm{~h} 30^{\prime}$ après injection de thymidine tritiée, dans le testicule droit de l'animal $\left(\hat{\sigma}_{2}\right)$ les spermatocytes I diplotènes de la fin du stade 2 du cycle suivant représentent le front de radioactivité (fig. B). Donc en 284 heures le front de radioactivité a parcouru un cycle entier et $28,8 \mathrm{p}$. roo du cycle suivant ; par suite la durée du cycle pour ce testicule est de 220 h $30^{\prime}$.

453 heures après injection, dans le testicule gauche de l'animal $\left(\hat{\sigma}_{2}\right)$ hémicastré, le front de radioactivité atteint les spermatides rondes de la fin du stade 8 du cycle suivant (fig. C), il a donc parcouru deux cycles entiers. Pour ce testicule la durée du cycle est de 226 h $30^{\prime}$.

$622 \mathrm{~h} 30^{\prime}$ après injection, dans le testicule droit de l'animal ( $\hat{\sigma}_{s}$ ) hémicastré le front de radioactivité atteint les spermatides allongées de la fin du stade 6 après avoir parcouru deux cycles et 74 p. Ioo du cycle suivant (fig. D). La durée du cycle pour ce testicule est de 227 heures.

\section{B. - Constance de la durée du cycle}

Pour les testicules appartenant à deux animaux nous avons trouvé des durées du cycle très voisines. La durée du cycle de l'épithélium séminifère apparaît par suite constante chez Macaca fascicularis (9,35 $\pm 0,27$ jours). Elle n'est affectée ni par l'hémicastration (après une semaine), ni par le traumatisme résultant de l'injection intratesticulaire de thymidine, ni par l'environnement.

\section{C. - Corollaire de la durée du cycle}

L'existence des associations rigoureuses de cellules germinales à une étape précise de leur évolution et à chaque stade du cycle nous permet en même temps d'estimer les durées des différentes phases de la méiose et de la spermiogenèse (cf. fig. I). Chez 
Macaca fascicularis la méiose complète dure $\mathrm{I} 4,4$ jours : la phase zygotène $(28 \mathrm{~h} 5)$ est relativement courte par rapport à la phase pachytène $(\mathrm{I} 64 \mathrm{~h})$. La spermiogenèse s'effectue en 13,7 jours.

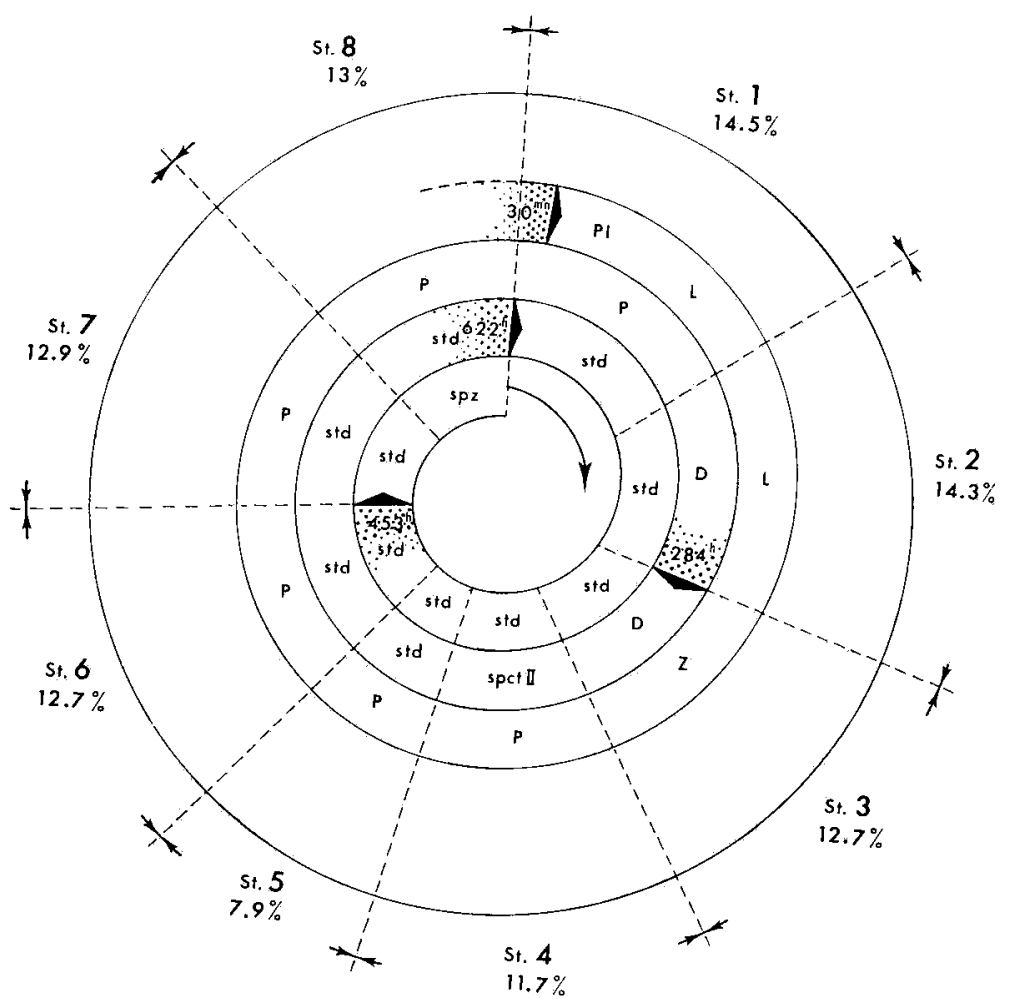

FıG. I. - Déplacement du front de vadioactivité au cours des stades du cycle de l'épithélium sêminifère

Sur ce diagramme les différentes générations de Spermatogonies n'ont pas été représentées St. : stade ; $\mathbf{P l}$ : spermatocyte primaire préleptotène ; $L$ : spermatocyte primaire leptotène ; $Z$ : spermatocyte primaire zygotène ; $\mathrm{P}:$ spermatocyte primaire pachytène ; $\mathrm{D}$ : spermatocyte primaire diplotène ; spct II : spermatocyte secondaire; std : spermatide ; spz : spermatozoïde.

\section{DISCUSSIONS}

La constance de la durée du cycle de l'épithélium séminifère pour une espèce de Mammifère donnée a déjà été notée par ORTAVANT (I958) travaillant sur des Béliers soumis à différentes photopériodes; par CLERMONT et HARVEY (1965) sur des rats normaux et hypophysectomisés; par DesciIN et ORTAVANT (I963) sur des Rats recevant de fortes doses d'androgènes ou d'hormones gonadotropes ; par CHOWDHURY et STEINBERGER (I964) sur des Rats subissant une destruction partielle du testicule ou une élévation de température; par DYM et CLERMONT (I968) sur des Rats soumis à une irradiation aux rayons $X$.

D'ailleurs la constance de la durée du cycle de l'épithélium séminifère est bien liée à celles de la fréquence relative des stades du cycle. 
Il semble que la longue durée du cycle de l'épithélium séminifère de l'Homme ( $\mathrm{r} 6$ jours) soit un caractère propre aux Hominiens et non aux Primates dans leur ensemble puisque la durée du cycle du Macaque crabier Macaca fascicularis ( 9,3 jours) est inférieure à celle du rat, I2 jours (CLERMONT et al., r959) et du Taureau, I3,5 jours (HOCHEREAU et al., I964).

Reçu pour publication en mars 1971.

\section{SUMMARY}

\section{DURATION OF THE SEMINIFEROUS EPITHELIUM CYCLE IN MACACA FASCICULARIS}

The germinal cells of Macaca fascicularis were labeled by means of tritiated thymidine injections in order to estimate the duration of the seminiferous epithelium cycle. It was 9.3 days, irrespective of hemicastfration.

The duration of the seminiferous epithelium cycle in man, viz. I6 days (HELLER and CLERMONT, I963) is the longest as compared to the values obtained by the same technique in other mammals. As the duration of the cycle in Macaca fascicularis is shorter than that of the rat : I 2 days (CLERMONT et al., I959) or the bull : I3,5 days (Hochereau et al., I964), the I6 day cycle seems typical of man and not of all primates.

\section{RÉFÉRENCES BIBLIOGRAPHIQUES}

Arsenieva N. A., Dubinin N. P., Orlova N. N., Bakulina E. D., ig6r. A radiation analysis of the duration of meiosis phases in the spermatogenesis of Macaca mulatta (russe). Dokl. Akad. Nauk. S. S. S. R., 141, $1486-I 489$.

Chowdhury A. W., Steinberger E., I 964 . A quantitative study of the effect of heat on germinal epithelium of Rat testis. Amer. J. Anat., 115, 509-524.

Clermont X., Harvey S. C., 1965. Duration of the cycle of the seminiferous epithelium of normal, hypophysectomized and hypophysectomized hormone treated albino-rats. Endocrinology, 76, 80-89.

Clermont Y., Leblond C. P., Messier B., r959. Durée du cycle de l'épithélium séminal du Rat. Arch. Anat. Micro. Morph. Exp., 48 bis, 37-54.

DANG D. C., I97I. Stades du cycle de l'épithélium séminifère du singe crabier Macaca fascicularis. Ann. Biol. anim. Bioch. Biophys., 11, 363-371.

Deschin J., Ortavant R., 1963. Influence des hormones gonadotropes sur la duree des processus spermatogénétiques chez le Rat. Ann. Biol. anim. Bioch. Biophys., 3, 329-342.

Dym M., Clermont Y., 1967. Cité par Clermont. Arch. Anat. Micro. Morph. Exp., 56 (supp. 3-4), $7-56$.

Heller C. G., Clermont Y., I963. Spermatogenesis in Man : an estimate of its duration. Science, $140,184-185$.

Hochereau M.-T., Courot M., Ortavant R., 1964. Marquage des cellules germinales du Bélier et du Taureau par injection de thymidine tritiée dans l'artère spermatique. Ann. Biol. anim. Bioch. Biophys., 4, 157-I6I.

Kopriwa B. M., Leblond C. P., rg62. Improvements in the coating technique of radioautography. J. Histochem. Cytochem., 10, 269-284.

Leblond C. P., Clermont Y., I952. Definition of the stages of the cycle of the seminiferous epithelium of the Rat. Ann. N. Y. Acad. Sci., 55, 548-573.

Ortavant R., I968. Le cycle spermatogénétique chez le Bélier. Thèse Fac. Sci. Paris. 


\section{PLANCHE I}

Autoradiographie testiculaire après injection de thymidine tritiée FIG. A

30-45 mn après injection, les spermatocytes primaires au stade préleptotène sont marqués (Stade I).

FIG. B

284 heures (ou II,8 jours) après injection, les spermatocytes primaires au stade diplotène sont marqués (fin Stade 2 ).

FIG. C

453 heures (ou I8,9 jours) après injection, les spermatides rondes sont marquées (Stade 8 ).

FIG. D

622 heures (ou 25,9 jours) après injection, les spermatides allongées sont marquées (Stade 6). 


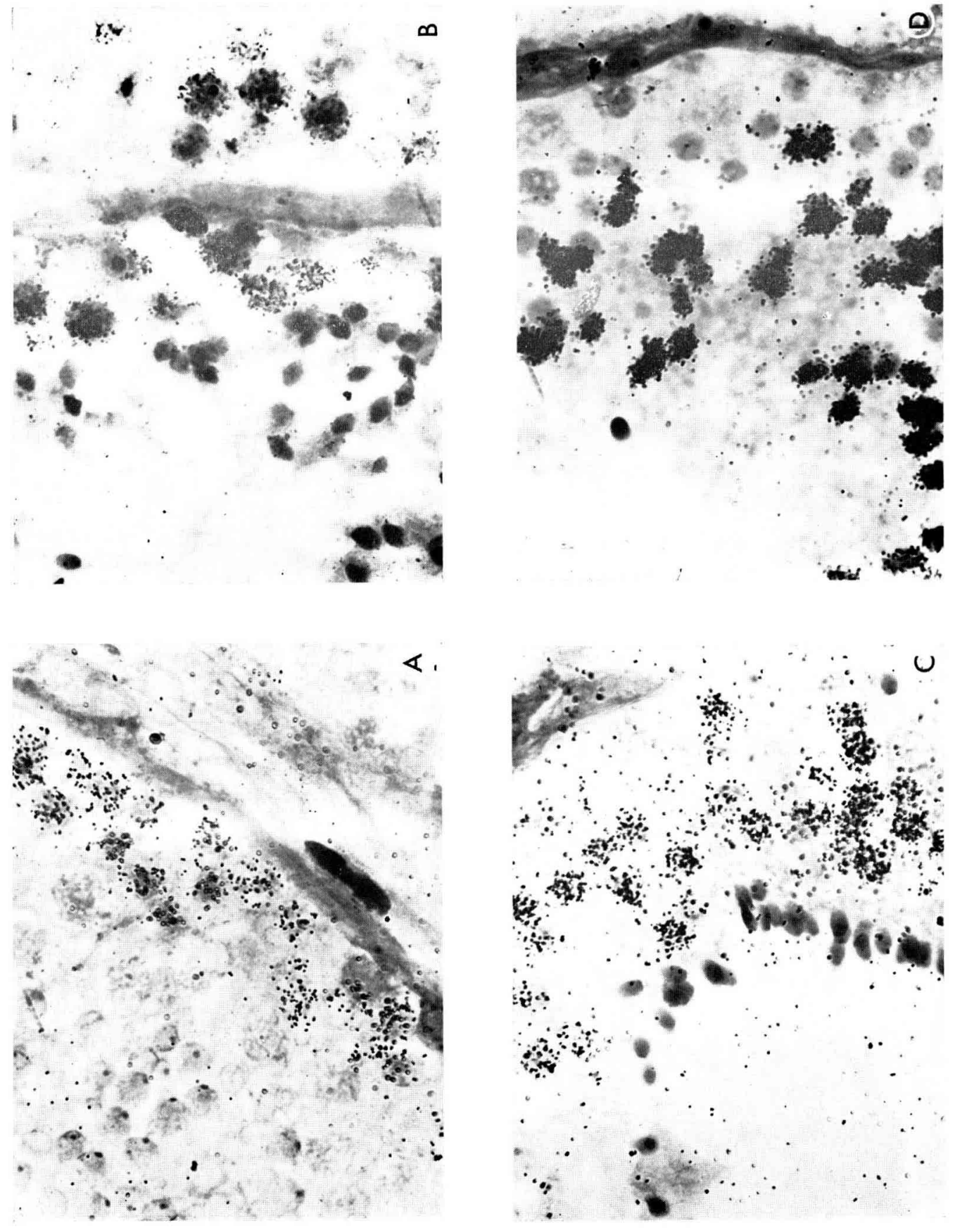

